\title{
Referential Communication by Chimpanzees (Pan troglodytes)
}

\author{
David A. Leavens \\ University of Georgia, Yerkes National Primate Research \\ Center, and University of Sussex
}

\author{
William D. Hopkins \\ Berry College and Yerkes National Primate Research Center
}

Roger K. Thomas
University of Georgia

\begin{abstract}
Two experiments were conducted to assess the referential function of chimpanzee (Pan troglodytes) gestures to obtain food. The chimpanzees received 1 trial per condition. In Experiment $1(N=101)$, in full view of the chimpanzee, a banana was placed on top of 1 of 2 inverted buckets or was hidden underneath 1 of the buckets. In Experiment $2(N=35), 4$ conditions were presented in constant order: (a) no food, no observer; (b) no food, observer present; (c) food present, no observer; and (d) food present, observer present. Gestures and visual orienting were used socially and referentially. The capacity for nonverbal reference may predate the Hominidae-Pongidae split, and the development of nonverbal reference may be independent of human species-specific adaptations for speech.
\end{abstract}

Referential signaling has been historically viewed as being uniquely human (Werner \& Kaplan, 1963). Referential signaling, as defined here, means the capacity to direct the attention of an observer to a distal object or entity. In linguistic reference, a symbol represents a concept, and through shared lexicons people can direct the attention of their conversational partners to these conceptual entities. Nonverbal reference, as the term is used in comparative research (e.g., Call \& Tomasello, 1994; Leavens \& Hopkins, 1998) and human developmental research (e.g., Adamson, 1996), is the capacity of a signaler to direct the attention of an observer through deictic and iconic manual gestures, the signaler's visual orienting behavior, and attention-directing tactile or auditory signals.

David A. Leavens, Department of Psychology, University of Georgia; Division of Psychobiology, Yerkes National Primate Research Center, Atlanta, Georgia; and Psychology Department, School of Life Sciences, University of Sussex, East Sussex, England. William D. Hopkins, Department of Psychology, Berry College, and Division of Psychobiology, Yerkes National Primate Research Center. Roger K. Thomas, Department of Psychology, University of Georgia.

This research was supported by National Institutes of Health Grants RR-00165 and NS-29574. Thanks to Dawn Pilcher for invaluable assistance with data collection. Thanks to J. Allen, Irwin Bernstein, Dorothy Fragaszy, Janet Frick, and B. E. Mulligan at the University of Georgia for helpful discussion of these data and for suggesting Experiment 2. Thanks to Tony Stubbens, University of Sussex, for technical assistance. Logistical support was provided by Frans de Waal and Darren Long at The Living Links Center for the Advanced Study of Ape and Human Evolution, Yerkes National Primate Research Center. Special thanks to Kim A. Bard, University of Portsmouth, for helpful commentary and critique. Thanks to Beena Khurana, University of Sussex, for helpful discussion of these findings.

Correspondence concerning this article should be addressed to David A. Leavens, Psychology Department, School of Life Sciences, University of Sussex, Falmer, East Sussex BN1 9QH, England. E-mail: davidl@ sussex.ac.uk
Pointing is the paradigmatic act of nonverbal reference (Bates, O'Connell, \& Shore, 1987). It has been claimed (a) that apes do not point (Corballis, 1991); (b) that they do not point with the index finger unless explicitly trained to point (Povinelli \& Davis, 1994); and (c) that compared with humans, when apes point, they do so with little understanding that pointing gestures influence the "internal mental states" of the observer (Povinelli, Bering, \& Giambrone, 2000, pp. 527-536). It has also been said that apes do not point while interacting with conspecifics (e.g., Povinelli et al., 2000). However, pointing among apes has been reported in captivity by de Waal (1982) and Savage-Rumbaugh (1986), among others, and in the wild by Inoue-Nakamura and Matsuzawa (1997) and Veà and Sabater-Pi (1998). Nevertheless, pointing behavior does not seem to be as common an occurrence among wild apes as it is among humans: Long-term field studies of chimpanzees in their natural habitats have not identified pointing as a frequent constituent of their communicative repertoires.

However, in captive settings, pointing is frequently exhibited by apes interacting with humans (e.g., Call \& Tomasello, 1994; Krause \& Fouts, 1997; Leavens \& Hopkins, 1998; Leavens, Hopkins, \& Bard, 1996; C. R. Menzel, 1999; Miles, 1990; SavageRumbaugh, 1986). Pointing is not limited to apes that have been language trained or raised as part of a human family unit (cf. Tomasello \& Call, 1997), and most reports of pointing by apes in captivity describe it as emerging "spontaneously," in the absence of explicit training (Call \& Tomasello, 1994).

Apes in captivity usually point with their whole hands, rather than with their index fingers (de Waal, 1982; Leavens \& Hopkins, 1999), except for language-trained apes, which frequently do point with the index finger (Call \& Tomasello, 1994; Krause \& Fouts, 1997); although less frequently, index finger pointing has been seen in apes that have not been language trained (Leavens \& Hopkins, 1998, 1999; Leavens et al., 1996). Perhaps because of this, it has been argued that apes do not point to distal objects, although they may exhibit "reaches" for unreachable food (e.g., 
Povinelli \& Davis, 1994). It is reasonable to ask whether apes, after a lifetime of experience in captivity, will continue to attempt to reach for unreachable food or whether these putative reaches might be better characterized as referential gestures. These questions can be answered by presenting unreachable food in the absence and presence of an observer. If such reaches constitute attempts to grasp unreachable food, then apes should be expected to display these behaviors irrespective of the presence or absence of a human observer. Alternatively, if these putative reaches constitute communicative gestures, then they should be displayed more frequently in the presence of an observer. This kind of "audience effect" has been demonstrated in previous laboratory studies. For example, Call and Tomasello (1994) demonstrated an effect of the presence of a human observer on the frequency of pointing with the index finger and with the whole hand by 2 orangutans. A similar audience effect on pointing was evinced by 3 chimpanzees (Leavens et al., 1996). Because of the small samples, it is difficult to generalize such findings to captive apes; for example, 1 of the subjects in the study by Leavens et al. (1996) was selected on the basis of his frequent, apparent pointing behavior. Recently, Hostetter, Cantero, and Hopkins (2001) demonstrated a strong influence of observer presence on gestural production in 49 chimpanzees; however, they did not distinguish among the different types of gestures exhibited.

In Experiment 1, the function of putative gestures in a sample of 101 chimpanzees was investigated by comparing the frequency of gestures associated with an unreachable banana in the presence and absence of a human observer. Experiment 1 was designed to answer two additional questions. First, would chimpanzees adjust their communicative behaviors according to whether an observer could see a banana and knew the banana's location (visible condition) or whether an observer did not see the banana when it was hidden and, therefore, was ignorant of the banana's location (hidden condition)? Hare, Call, Agnetta, and Tomasello (2000) have shown that chimpanzees discriminate between different states of observers' visual access to food location in a competitive context, and we wanted to determine whether such discriminations would be exhibited in a noncompetitive context.

The second question addressed in Experiment 1 was the extent to which chimpanzees' gestures and visual orienting behavior might be referential; that is, how might the location of a banana, both visible and hidden, influence the spatial deployment of such behavior? Previous studies in which apes' pointing has been shown to correlate with object location, in the context of hidden food or tools, are few in number and are characterized by small samples of 1 to 4 apes (Call \& Tomasello, 1994; Krause \& Fouts, 1997; C. R. Menzel, 1999; Woodruff \& Premack, 1979). Experiment 1 was designed to test the generality of the previous findings.

Because previous studies used experimental conditions in which food presence was a constant factor while observer presence was manipulated (e.g., Call \& Tomasello, 1994; Krause \& Fouts, 1997; Leavens et al., 1996), Experiment 2, using 35 chimpanzees, was designed to investigate the effects of both food and observer. Baseline rate of response was determined in four conditions administered in constant order: (a) no food, no observer; (b) no food, observer present; (c) food present, no observer; and (d) food present, observer present. Such data should clarify, for example, how long it takes for chimpanzees to mobilize arm extensions and whether these are mistakenly interpreted as being communi- cative when an experimenter arrives coincidently with these arm extensions.

\section{Experiment 1}

\section{Method}

Subjects. Subjects were 101 chimpanzees (Pan troglodytes; 48 males, 53 females) housed at the Yerkes National Primate Research Center (YNPRC) at Emory University. To our knowledge, these chimpanzees had not received language training. All subjects were treated in accordance with the ethical standards of the American Psychological Association (1992). YNPRC is fully accredited by the American Association for Laboratory Animal Care. No animal was food deprived in the course of the experiments reported in this study.

Materials. Two experimenters participated in each trial. Each subject received one each of two trial types (conditions), in randomized order. Two identical plastic buckets were inverted and placed approximately $70 \mathrm{~cm}$ from the subjects' cages, approximately $1.5 \mathrm{~m}$ apart (see Figure 1). All trials were videotaped to enable the computation of reliability estimates.

Procedure. Experimenter 1 placed a banana either on top of an inverted bucket (visible condition) or beneath an inverted bucket (hidden condition), according to a predetermined, randomized schedule. Experimenter 1 then started the video camera and departed. Experimenter $1 \mathrm{did}$ not communicate the location of the banana to Experimenter 2. Subsequently (at least $30 \mathrm{~s}$ later), Experimenter 2 arrived, centered himself between the buckets, and attempted to engage the visual attention of the subject by looking at the subject and speaking the subject's name.

In the visible condition, Experimenter 2 responded to any apparent communicative behavior, as defined in Table 1, by delivering the banana to the subject. Every subject was given at least $30 \mathrm{~s}$ to respond. Bananas were delivered on response or at the termination of the trial. In the hidden condition, when both subject and experimenter were visually engaged with each other, Experimenter 2 attempted to determine which bucket concealed the banana via the gestures and postures of the subject. If Experimenter 2 identified the bucket hiding the banana, the banana was given immediately to the subject. If the bucket did not contain the banana, then Experimenter 2 immediately overturned the remaining bucket and delivered the banana to the subject. If no obvious communicative gesture was exhibited within a minimum interval of $30 \mathrm{~s}$ after his arrival, Experimenter 2 overturned one or both buckets and delivered the banana, when found, to the subject. After

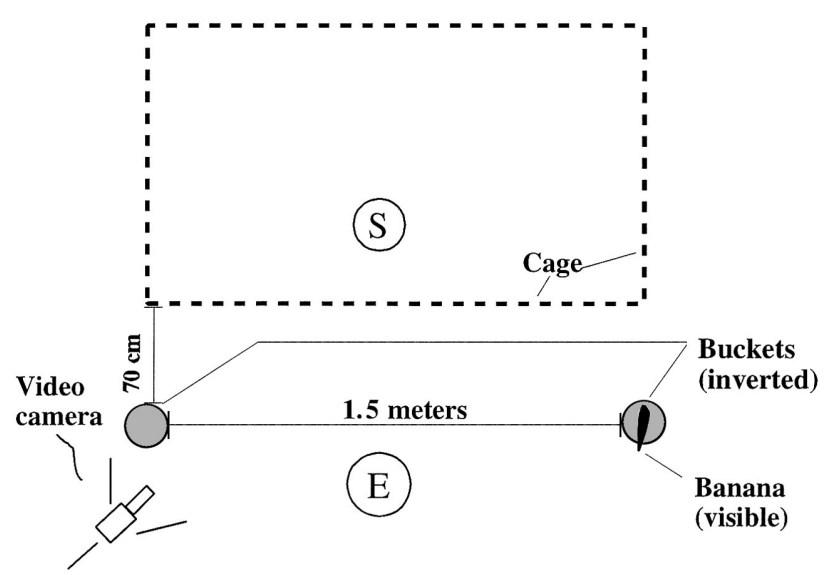

Figure 1. Setup for the experiment on the effects of hidden food on gestural production. The visible condition is depicted, in which a banana was placed on the inverted bottom of a plastic bucket. In the hidden condition, a banana was placed beneath an inverted bucket. S represents the subject, and E the experimenter. Drawing is not to scale. 
Table 1

Distribution of Gesture Type With Gaze Alternation (GA) and Without Gaze Alternation (NO GA) Between Bucket and Experimenter 2 by Condition in Experiment 1

\begin{tabular}{|c|c|c|c|c|}
\hline \multirow[b]{2}{*}{ Gesture type } & \multicolumn{2}{|c|}{$\begin{array}{l}\text { Visible } \\
\text { condition }\end{array}$} & \multicolumn{2}{|c|}{$\begin{array}{l}\text { Hidden } \\
\text { condition }\end{array}$} \\
\hline & NO GA & GA & NO GA & GA \\
\hline \multicolumn{5}{|l|}{ Observer directed } \\
\hline Beg & 4 & 17 & 3 & 14 \\
\hline Hold hand out & 3 & 16 & 4 & 13 \\
\hline \multicolumn{5}{|l|}{ Object directed } \\
\hline Beg (directed at bucket) & 1 & 2 & 0 & 4 \\
\hline Point with whole hand & 2 & 20 & 4 & 19 \\
\hline Point with index finger & 0 & 3 & 0 & 5 \\
\hline Other & 1 & 7 & 0 & 7 \\
\hline No gesture & 9 & 16 & 10 & 18 \\
\hline Total & 20 & 81 & 21 & 80 \\
\hline
\end{tabular}

Note. $\quad N=101$.

delivery of the banana, Experimenter 2 turned off the video camera and departed. Each subject received only one trial in each condition; thus, all analyses were based on first-trial data.

Experimenter 2 recorded (a) whether the subject gestured, (b) gesture type, (c) whether the subject vocalized, and (d) whether the subject looked successively between Experimenter 2 and one or both buckets. If subjects looked between the experimenter and both buckets, Experimenter 2 recorded the bucket to which the preponderance of gaze was directed as a result of successive visual orienting between Experimenter 2 and this bucket.

Behavioral measures. Gestures are listed in Table 1. A distinction is made in Table 1 between object-directed and observer-directed gestures. In a horizontal plane, parallel to the floor, a judgment was made with respect to whether the orientation of a putative gesture was more toward the experimenter, toward one of the buckets, or elsewhere. In the vertical plane, perpendicular to the floor and parallel to the cage mesh bordering the corridor, arm or hand orientations deemed to be directed within approximately $20^{\circ}$ above or below either the banana or the experimenter's thorax were categorized as being banana directed or experimenter directed, respectively. Only the first manual gesture, if any, exhibited by any given subject was recorded for analysis.

In addition to gestural responses, visual orienting behavior was recorded dichotomously as either constituting successive looking between one of the buckets (or banana) and Experimenter 2 (gaze alternation) or not constituting successive looking between one of the buckets (or banana) and Experimenter 2. When multiple gaze alternation occurred, Experimenter 2 recorded the preponderant orientation of the gaze. Vocalizations were recorded as present or absent.

Data analysis. Because the data reported are at nominal level of measurement, nonparametric tests, including binomial tests and chi-square statistics, were used. All tests were two-tailed.

Reliability. Reliability estimates were derived by comparing the data sheets from the live coding, for which David A. Leavens and William D. Hopkins served as observers, and a later viewing of the videotapes by David A. Leavens. Because not every subject stayed within camera view throughout the observation periods, only a subset of trials were available for reliability estimates. Only trials in which the subject was visible on the videotaped record from the time Experimenter 2 arrived to the time the subject was reinforced were used. Including visible and hidden trials, there were 91 trials ( $45 \%$ of all 202 trials) that met this criterion. In the case of disagreements, the data from the videotaped record were used. Cohen's $\kappa=.73$, for whether subjects gestured; Cohen's $\kappa=.82$, for whether subjects exhibited gaze alternation; and Cohen's $\kappa=.75$, for whether subjects vocalized. Cohen's kappa is a reliability estimate that corrects for agreement by random chance. Kappa values $>.70$ are considered excellent (Bakeman \& Gottman, 1986).

\section{Results}

Tests for influence of observer presence. Because the chimpanzees were not restrained and could move in and out of camera range, not all gestures were captured on film. Assessment of the influence of observer presence was limited to those chimpanzees who gestured on camera within $30 \mathrm{~s}$ before and after the arrival of Experimenter 2. Of the 101 chimpanzees, 50 gestured on camera within $30 \mathrm{~s}$ of the arrival of Experimenter 2 in the visible condition, and 51 gestured on camera within $30 \mathrm{~s}$ in the hidden condition (62 subjects gestured in one or both conditions). Figure 2 depicts the temporal distribution of these gestures. Gestural production was significantly associated with the presence of the experimenter: visible condition, binomial test, $z(49)=4.81, p<.0001$; hidden condition, binomial test, $z(50)=5.60, p<.0001$. All gestures exhibited prior to the arrival of Experimenter 2 were conservatively coded as having occurred in the absence of the observer for these tests. With one exception, the 101 gestures exhibited by 62 chimpanzees occurred exclusively during the approach or after the arrival of Experimenter 2.

Tests for an influence of condition. To statistically compare the effects of hiding food, we selected subjects that met two criteria: (a) Either they gestured within $30 \mathrm{~s}$ of the arrival of Experimenter 2 or they did not respond, and (b) they were observed during both the visible and hidden conditions. Sixty-eight chimpanzees met both criteria. Systematic statistical tests revealed no influence of test order (i.e., whether the subjects experienced the visible or hidden condition first) on propensity to gesture, vocalize, exhibit gaze alternation, or gesture toward the buckets. Test order was therefore not considered in subsequent analyses.

There was no apparent influence of hiding the food on subjects' propensity to gesture (binomial test, $n=14, n s$ ). Hence, there was no evidence that the chimpanzees exhibited different propensities to gesture in accordance with whether the banana was visible or hidden.

Because the chimpanzees were tested in their home cages with cage mates present, we asked whether subjects that had opportunities to see cage mates experiencing the experiment prior to their own participation might gesture more frequently. The $101 \mathrm{chim}-$ panzees were categorized as follows: (a) the 1st in a specific cage to experience the experiment $(n=38)$, (b) the 2 nd to experience the experiment $(n=31)$, and (c) the 3rd-8th subjects to participate in the experiment $(n=32)$. The percentages of chimpanzees that were $1 \mathrm{st}, 2 \mathrm{nd}$, and $3 \mathrm{rd}-8 \mathrm{th}$ and that gestured at least once (in either the visible or hidden condition, or both) were $84 \%, 74 \%$, and $66 \%$, respectively, $\chi^{2}(2, N=101)=3.25, n s$. Thus, there was no apparent facilitatory effect of observing the experiments on gestural production.

The number of chimpanzees that increased the specificity of their gestures when the food was out of view (i.e., gestured toward Experimenter 2 in the visible condition and toward a bucket in the hidden condition, $n=7$ ) was compared with the number of subjects who decreased the specificity of their gestures when the food was hidden (i.e., gestured toward a bucket in the visible 
A

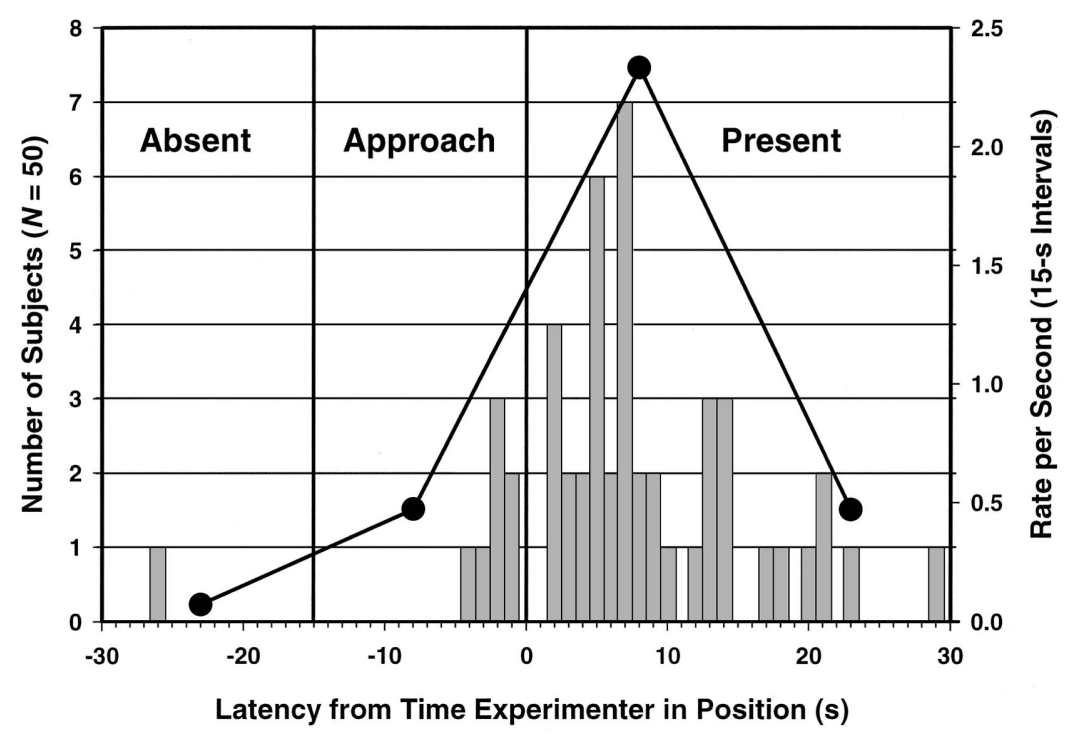

B

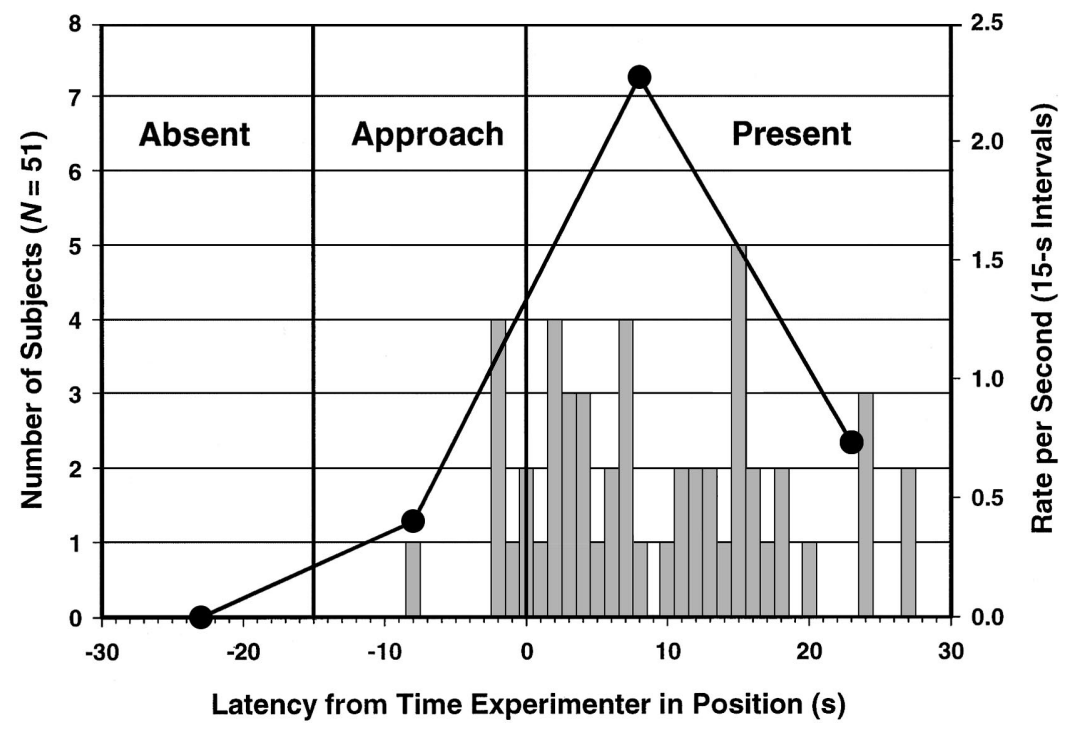

Figure 2. Temporal distribution of gestures exhibited by chimpanzees. The second experimenter arrived and centered himself in front of each subject's cage at 0 . All subjects were observed for a minimum of $30 \mathrm{~s}$ prior to the arrival of the second experimenter. The duration of the approach phase varied across subjects because of the different distances of subjects' cages from the exits; the maximum duration of the approach phase is depicted here. Vertical bars denote gestures, and circles represent the rate of gestural production in 15-s blocks. A: Results from the visible condition. B: Results from the hidden condition. The terms Absent, Approach, and Present refer to the status of the second experimenter.

condition and toward Experimenter 2 in the hidden condition, $n=$ 5). There was no apparent influence of hiding the food on the specificity of subjects' gestures (binomial test, $n=12, n s$ ).

There was no apparent influence of hiding the food on subjects' propensity to vocalize (binomial test, $n=14, n s$ ). With respect to gaze alternation between a bucket and the experimenter, there was no apparent influence of hiding the banana on the propensity to exhibit gaze alternation between a bucket and the experimenter (binomial test, $n=22, n s$ ).
Tests to assess the referential function of gestures and gaze. For these analyses, all of the chimpanzees that gestured were included. In the visible and hidden conditions, 53\% and $47 \%$, respectively, of the gestures were directed at the experimenter, and $37 \%$ and $44 \%$, respectively, were directed toward the buckets. The remainder in both conditions were either directed elsewhere or involved postural, rather than manual, orientations (see Table 1). In the visible and hidden conditions, respectively, 28 and 32 chimpanzees gestured toward one of the two buckets, gesturing 
significantly more often to the baited as opposed to the unbaited buckets in both conditions: visible, 27 to baited bucket, 1 to unbaited bucket, binomial test, $z(27)=4.91, p<.001$; hidden, 24 to baited bucket, 7 to unbaited bucket, binomial test, $z(30)=3.05$, $p<.01$ (the target location of the whole hand point exhibited by 1 subject in the hidden condition was not discernable). The location of the banana thus determined the orientation of these gestures; that is, they were referential in function.

Gaze alternation, defined as looking successively between Experimenter 2 and the banana or baited bucket, was significantly associated with the display of gestures in both the visible and hidden conditions, as depicted in Figure 3 (see also Table 1): visible condition, binomial test, $z(100)=6.07, p<.001$; hidden condition, binomial test, $z(100)=6.07, p<.001$. Chimpanzees that gestured were more likely to exhibit gaze alternation than those that did not gesture.

Tests of communicative efficacy when a banana was hidden. In the hidden condition, data on performance of the observer in selecting the correct (baited) bucket were available for 97 trials; the experimenters inadvertently failed to record which bucket was overturned on 4 trials. Experimenter 2 selected the correct bucket on 69 of the 97 trials $(71 \%$ correct, $p<.01$ ). Chimpanzees communicated effectively about hidden food, even when given only one opportunity to respond.

That the chimpanzees were tested in their home cages raises the question of whether the chimpanzees might have learned to exhibit clearer communicative behavior as a result of watching trials being administered to their cage mates. To assess whether the opportunity to observe trials administered to a cage mate influenced performance in the hidden condition, we categorized subjects as being the 1 st $(n=37)$, 2nd $(n=30)$, or 3 rd- 8 th $(n=30)$ subject in a cage to receive the task. Performances were $73 \%, 73 \%$, and $63 \%$, respectively, $\chi^{2}(2, N=97)=3.25, n s$. Therefore, having the opportunity to observe a cage mate did not improve performance in the hidden condition. Additionally, to assess whether performance was affected by the number of chimpanzees housed in a cage, we categorized subjects as being solitary $(n=7)$, having 1

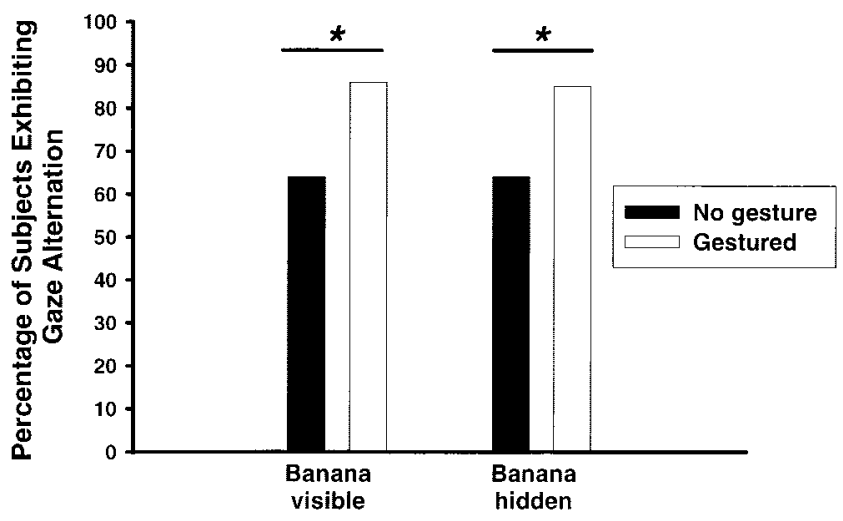

Figure 3. Gaze alternation between the buckets or banana and the experimenter was exhibited by significantly more chimpanzees that gestured than by chimpanzees that did not gesture, in both the visible and hidden conditions. In the visible condition, $25 \%$ did not gesture, and $75 \%$ gestured. In the hidden condition, $28 \%$ did not gesture, and $72 \%$ gestured. $* p<.001$. cage mate $(n=36)$, or having several cage mates (3-8 chimpanzees in a cage; $n=54$ ); performances were $71 \%, 72 \%$, and $70 \%$, respectively, $\chi^{2}(2, N=97)=0.04, n s$. Hence, numerically different opportunities to observe a cage mate had no apparent influence on individual performances.

\section{Discussion}

The chimpanzees in this study gestured overwhelmingly as a function of the imminent arrival or presence of an experimenter. This finding is consistent with earlier, small-sample studies with 2 to 3 apes (Call \& Tomasello, 1994; Leavens et al., 1996). Suggestions that the chimpanzees are reaching toward obviously unreachable food in the experimental conditions examined here are untenable in light of these results.

There was no apparent influence of hiding the banana on subjects' propensities to gesture, vocalize, or exhibit gaze alternation between the buckets and the second experimenter; nor was there any obvious influence of hiding the banana on subjects' gestural orientation.

The gestures and gaze-alternating behavior recorded in the present study were under the stimulus control of the banana's location. When these apes gestured somewhere other than at the experimenter, they overwhelmingly gestured toward the banana, whether visible or hidden. As reported previously (Leavens \& Hopkins, 1998), gaze alternation between the baited buckets and the experimenter was significantly associated with the production of gestures. Thus, visual orienting and gestural production are linked in chimpanzees, which suggests that there may be an ostensive aspect of gaze during gestural production (cf. Gómez, 1996).

The chimpanzees effectively communicated the location of a hidden banana to an experimenter who was ignorant of its location. This finding is equivocal insofar as there was inadequate control for inadvertent cuing in the present study. It is not possible, for example, to assert that it was the chimpanzees' visual orienting and gestural behaviors (only) to which the second experimenter responded during the hidden food trials and not their postures, spatial position in cage, behavior of cage mates, and so on. It is nonetheless clear that the chimpanzees frequently gestured and apportioned their visual orienting behavior nonrandomly with respect to both observer presence and the location of the hidden banana.

It might be that the audience effect reported here is attributable to endogenous periodicity in the mobilization of apparent gestural responses, which are then deployed only coincidentally with the arrival of Experimenter 2. There are also no published data, to our knowledge, on the baseline rates of apparent gestural production directed outside the subjects' cages in the absence of both food and an experimenter. To address these issues, we conducted the second experiment in which the presentation of food and an observer were manipulated.

\section{Experiment 2}

\section{Method}

Subjects. Subjects were 35 chimpanzees (20 males, 15 females) housed at the YNPRC.

Materials. The experimental arrangement is depicted in Figure 4. A video camera was placed to each subject's right, approximately $1.5 \mathrm{~m}$ from 
the cage (this was the maximum possible distance because of the width of the corridor), and oriented to encompass in the field of view as much as possible of the interior of each cage.

Procedure. Subjects were administered four conditions in invariant order: (a) no food, no experimenter; (b) no food, experimenter present; (c) food present, no experimenter; and (d) food present, experimenter present. Each sequence of four conditions constituted one trial. The duration of each condition varied across subjects (15-29 s) but was held to a minimum of $15 \mathrm{~s}$ for all subjects in all conditions. From a total experimental duration of $49.57 \mathrm{~min}$, the total time that chimpanzees were visible on tape was 36.52 min (i.e., over all four conditions, the 35 subjects were visible on film a total of $74 \%$ of the time).

The procedure was as follows: Experimenter 1 positioned the video camera and announced "start" as he retreated to the subject's right to a distance down the corridor at which he was no longer visible to the focal subject but clearly visible to Experimenter 2, who was staged down the same corridor to the subject's left, out of the subject's view. After a minimum interval of $15 \mathrm{~s}$ (Condition 1: no food, no experimenter), Experimenter 1 signaled Experimenter 2 with a hand signal to approach the cage. When Experimenter 2 reached the position indicated in Figure 4, he announced "here." Experimenter 2 looked at the subject, recording the subject's behavior on a coding sheet, but did not verbally respond to any apparent communicative behaviors. After a minimum of $15 \mathrm{~s} \mathrm{(Condition} \mathrm{2:}$ no food, experimenter present), Experimenter 2 departed while visually signaling Experimenter 1 to approach. Experimenter 1 (a) approached rapidly, (b) immediately placed the banana as indicated in Figure 4, and (c) departed rapidly. Although Experimenter 1 was briefly present during this condition to place the banana, he did not visually attend to the subject at any time. After at least $15 \mathrm{~s}$ had elapsed (Condition 3: food present, no experimenter), Experimenter 1 signaled Experimenter 2 to reapproach the cage. When Experimenter 2 arrived and again stood in the position indicated in Figure 4, he announced "here." After a minimum interval of $15 \mathrm{~s}$ (Condition 4: food present, experimenter present), Experimenter 1 announced "end." Immediately after the end signal, either Experimenter 1 or Experimenter 2 delivered the banana to the subject.

Behavioral measures. Behavioral measures were the same as in Experiment 1 (see Table 1), with the following modification: Visual orienting behavior during Condition 4 was defined dichotomously as gaze alternation present, when there was successive looking between the video camera

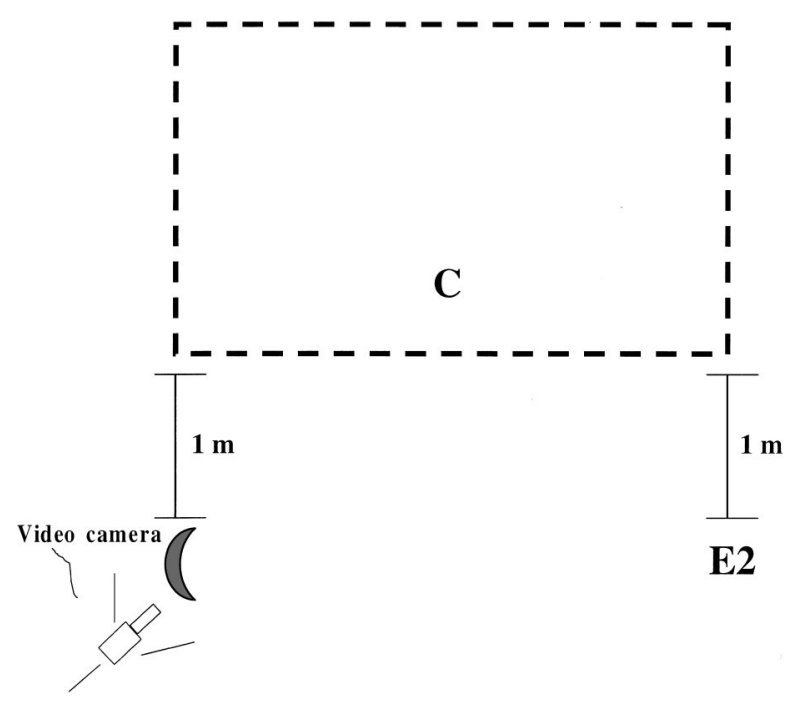

Figure 4. Experimental arrangement for Experiment 2. C represents the subject, and E2 the second experimenter. The gray crescent depicts a banana. Drawing is not to scale. or banana and Experimenter 2, and gaze alternation absent, when no successive visual orienting between the video camera or banana and Experimenter 2 was observed. Responses were recorded by Experimenter 2 on a data sheet during his presence in Conditions 2 and 4 . Independently, David A. Leavens, who was Experimenter 1 in all trials, coded these behaviors from the videotaped record.

Reliability. Reliability was assessed by comparing the observations from videotape (David A. Leavens) with those performed by Experimenter 2 (William D. Hopkins) in Conditions 2 and 4. Included in the reliability assessments were subjects that were visible on videotape throughout the condition of interest and subjects that were not visible on videotape for the entire duration of the condition of interest but for whom both observers agreed that the subject gestured (Conditions 2 and 4), vocalized (Condition 4 only), or exhibited gaze alternation between the banana and Experimenter 2 (Condition 4 only). In the case of disagreements, the data from the videotaped record were used. In Condition 2, reliability of gesture type, based on 23 subjects, was Cohen's $\kappa=.78$; the corresponding analysis for Condition 4, based on 16 subjects, was Cohen's $\kappa=.80$. On the basis of 23 subjects, agreement whether subjects alternated their gaze between the banana and Experimenter 2 in Condition 4 was $100 \%$.

Analyses. Cochran's $Q$ was used to test the null hypothesis that gestures were randomly distributed in time. This statistic is appropriate for data that can be dichotomously categorized over more than two levels of an independent variable that involves use of the same or related samples in each level (Siegel \& Castellan, 1988). All tests were two-tailed.

\section{Results}

Observer and food effects on the propensity to gesture. Of 35 subjects, 17 gestured in this study. Twelve subjects gestured on camera, and an additional 5 chimpanzees gestured off camera. Five gestures were recorded by Experimenter 2 (during Conditions 2 and 4) that were not observed on camera; so, it may be possible that other gestures may have occurred in the absence of Experimenter 2 that were also not caught on camera. Figure 5 depicts the temporal distribution of the first gestures by 11 subjects, plus a second gesture by 1 subject, Carl. Table 2 lists subjects that gestured and the conditions in which each subject gestured; this table includes gestures seen by Experimenter 2 but not on camera. Although this may bias against the null hypothesis, opposing this bias was a complementary observational bias in favor of the null hypothesis due to chimpanzees being visible on camera an average of $10 \mathrm{~s}$ longer when Experimenter 2 was absent than when Experimenter 2 was present. The chimpanzees in this study did not distribute their gestures randomly with respect to the four conditions, Cochran's $Q(3, N=17)=13.78, p<.05$. Inspection of Table 2 reveals that the majority of gestures (17 of 20) occurred in Conditions 2 and 4. Nearly twice as many gestures were initiated when both food and an experimenter were present compared with when the experimenter was present but food was absent (11 and 6, respectively). Thus, the chimpanzees gestured in the presence of an observer (Experimenter 2) and typically did not gesture in the absence of an observer, irrespective of whether food was present. Inspection of Figure 5 reveals that the three gestures exhibited in the absence of an observer were displayed in the $2 \mathrm{~s}$ prior to the arrival of Experimenter 2 (that is, on the imminent approach of the observer).

Gaze alternation. Gaze alternation between the banana and the experimenter could be assessed only in Condition 4. There was a significant association between subjects' propensity to gesture and to exhibit gaze alternation between the experimenter and the food, 

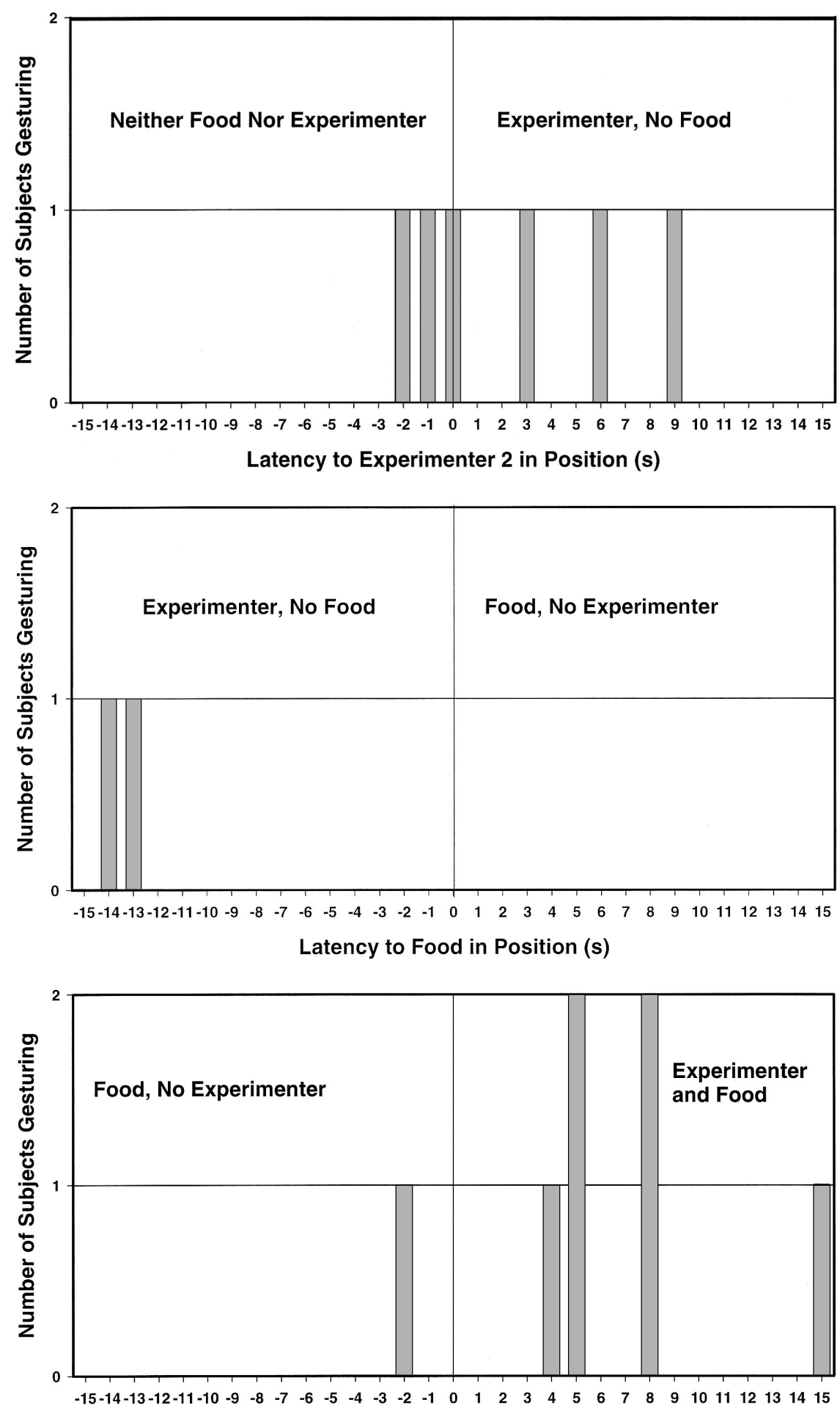

Latency to Experimenter 2 in Position (s)

Figure 5. Distribution of gestures with respect to the time Experimenter 2 or a banana was fully in position during each of the three transitions in Experiment 2. Top: Transition from Condition 1 to Condition 2. Middle: Transition from Condition 2 to Condition 3. Bottom: Transition from Condition 3 to Condition 4. One subject, Carl, is represented twice, at $0 \mathrm{~s}$ in the top panel and at $4 \mathrm{~s}$ in the bottom panel. 
Table 2

Subjects Who Gestured in Experiment 2

\begin{tabular}{llllll}
\hline & \multicolumn{5}{c}{ Condition } \\
\cline { 2 - 5 } \multicolumn{1}{c}{ Subject } & 1 & 2 & 3 & 4 & Total \\
\cline { 2 - 5 } Boisfeullet $^{\mathrm{a}}$ & 0 & 1 & 0 & 0 & 1 \\
Callie $^{\mathrm{a}}$ & 0 & 0 & 0 & 1 & 1 \\
Reba $^{\mathrm{a}}$ & 0 & 0 & 0 & 1 & 1 \\
Ossabaw $^{\mathrm{a}}$ & 0 & 0 & 0 & 1 & 1 \\
Cheetah $^{\mathrm{a}}$ & 0 & 0 & 0 & 1 & 1 \\
Lucy & $1^{\mathrm{b}}$ & $1^{\mathrm{b}}$ & 0 & 0 & 2 \\
Carl & 0 & $1^{\mathrm{b}}$ & 0 & $1^{\mathrm{b}}$ & 2 \\
Merv & 0 & $1^{\mathrm{b}}$ & 0 & 0 & 1 \\
Duncan & $1^{\mathrm{b}}$ & 0 & 0 & 0 & 1 \\
Brodie & 0 & $1^{\mathrm{b}}$ & 0 & 0 & 1 \\
Ellie & 0 & 0 & $1^{\mathrm{b}}$ & $1^{\mathrm{b}}$ & 2 \\
Clint & 0 & 0 & 0 & $1^{\mathrm{b}}$ & 1 \\
Amanda & 0 & $1^{\mathrm{c}}$ & 0 & 0 & 1 \\
Anna & 0 & 0 & 0 & $1^{\mathrm{c}}$ & 1 \\
Winston & 0 & 0 & 0 & $1^{\mathrm{c}}$ & 1 \\
Puddin' & 0 & 0 & 0 & $1^{\mathrm{c}}$ & 1 \\
Storer & 0 & 0 & 0 & $1^{\mathrm{c}}$ & 1 \\
\multicolumn{1}{c}{ Total } & 2 & 6 & 1 & 11 & 20 \\
\hline
\end{tabular}

Note. 0s indicate that no gesture was observed, and $1 \mathrm{~s}$ indicate that a gesture was observed.

${ }^{\text {a }}$ These subjects were visible on camera throughout all four conditions. ${ }^{b}$ These gestures were recorded on camera. ${ }^{c}$ These gestures were observed by Experimenter 2 but not recorded on camera.

$\chi^{2}(1, N=35)=5.45, p<.05$, which is consistent with previous reports (Leavens \& Hopkins, 1998) and with data reported in Experiment 1 . Twelve of the 24 subjects $(50 \%)$ that did not gesture in Condition 4 did exhibit gaze alternation between the banana and the experimenter; whereas 10 of the 11 subjects (91\%) that gestured in Condition 4 also exhibited gaze alternation between the experimenter and the banana.

\section{Discussion}

The data indicate that gestural production was related to the impending arrival or presence of a human observer in the present experiment (Figure 5). The data also demonstrate an association between visual orienting behavior and gestural production in chimpanzees, as reported in previous studies (Krause \& Fouts, 1997; Leavens \& Hopkins, 1998; Leavens et al., 1996) and in Experiment 1. Thus, the audience effect in Experiment 1 does not seem to be attributable to endogenous temporal patterns of gestural production. The chimpanzees' gestural production in Experiment 2 was influenced by the presence and absence of the experimenter. There does not appear to be any meaningful difference in rates of gestural production between the condition in which neither food nor an experimenter was present and the condition in which food was present but the experimenter was absent. In both cases, gestural production was rare. Gestural production in the presence of unreachable food is comparable to gestural production in the baseline condition in which no food or observer was present.

\section{General Discussion}

The audience effects in Experiments 1 and 2 refute claims that chimpanzees, even after a lifetime in captivity, attempt to reach for obviously unreachable food. Gestures of all types were deployed only on the imminent approach or after the arrival of an experimenter; this pattern was seen in both experiments (see Figures 2 and 5). Hence, all of these gestures appear to be communicative in function. When gestures were directed toward one of the two buckets, they were overwhelmingly directed toward the baited bucket. Such directed gestures constitute acts of pointing for the chimpanzees in this colony. Similar results were obtained in the analyses of visual orienting behavior. Thus, the deployment of gestures and gaze alternation between a banana and an observer were manifested as integrated patterns of nonverbal reference. Because all data here are first-trial data, the chimpanzees had acquired these response patterns prior to the administration of the present experiments.

There is a large and growing literature on the cognitive implications of communicative pointing by human infants in late infancy, that is, pointing with concomitant gaze alternation between the target of the point and an observer (e.g., Butterworth, 2001; Tomasello, 1995). By Baron-Cohen's (1999) definition, intentional communication, of which pointing is the paradigmatic example, occurs only when the putative goal of the signaler is to influence the state of knowledge of an observer. On this account, intentional communication implies that an organism must be able to distinguish the behavioral correlates of different states of knowledge in an observer. Also on this account, pointing to request objects or, more generally, instrumental use of gestures does not constitute intentional communication. This approach to intentional communication suffers from the inability to objectively verify these putative goals in the signalers; there is no independent measure of the communicative intent of a pointing infant or ape. It is an open question whether this inability to verify communicative intent is related to technological limitations (e.g., communicative "intentions" might be determinable if we had appropriate knowledge of brain activity during communicative episodes, cf. Bergmann, 1962) or to more general epistemological problems with models of cognition founded on mental causality in which mental states are held to be causal in behavior (e.g., Leavens, Hostetter, Wesley, \& Hopkins, 2004; Thompson, 1997). We favor an operational perspective on intentional communication; namely, these animals are communicating intentionally because they require an audience to exhibit the behavior and they exhibit a coordinated pattern of gestural and visual orienting behavior that is determined by the locations of both an observer and food. Thus, these gestures are demonstrably "about" specific items in their environments.

Hiding a banana beneath a bucket had no apparent influence on the propensity to gesture, the target of gestures (observer vs. buckets), propensity to vocalize, or propensity to alternate gaze between buckets and an observer. Had the chimpanzees exhibited reduced communicative behavior in the hidden condition, one might conclude that the sight of a banana had a potentiating effect on communication. Conversely, had they communicated more or with greater specificity (more toward the buckets than to the experimenter) in the hidden condition, one might conclude that they were adjusting their communication in accordance with the state of ignorance (or its behavioral correlates) of the second experimenter. The chimpanzees deployed the same kinds of communicative behavior whether the banana was visible or hidden; therefore, the chimpanzees perceived no particular difficulty in the hidden versus visible condition. 
Some accounts of the development of intentional signaling by human infants emphasize the paralinguistic or prelinguistic nature of these gestures (e.g., Baldwin, 1995). The present data from a large sample of non-language-trained chimpanzees with no explicit training to gesture to hidden food suggest that the ability for referential signaling (a) is not uniquely human and (b) does not require the neurobiological adaptations for speech unique to the human lineage (Leavens \& Hopkins, 1998, 1999). As E. W. Menzel (1973) noted, "One good reason that chimpanzees very seldom point manually is that they do not have to" (p. 218, italics in original); in other words, for apes in the wild, the behavioral correlates of visual attention, including posture and head orientation, are sufficient to inform an observer as to the direction of a conspecific's apparent interest. There are strong similarities in the circumstances in which preverbal human infants and captive apes gesture referentially: (a) Both human infants and captive apes experience barriers to grasping desirable objects directly; for human infants there are limitations on their locomotor capacities and for captive apes there is cage mesh or bars, and (b) both human infants and captive apes experience histories of profligate food provisioning by adult caretakers (Leavens et al., 1996). Thus, most apes or humans under these circumstances may develop referential gestures through autoshaping, incidental operant conditioning, or imitation. The present data constrain theories regarding the evolution of human communication. In contrast to some claims (Baron-Cohen, 1999), the capacity for referential signaling appears to be a synapomorphic (shared, derived) trait within Hominoidea (Leavens \& Hopkins, 1998; Leavens et al., 1996). The present data, together with several lines of convergent evidence, render more plausible theories of the gestural origins of language (Corballis, 1991). From a behavioral perspective, there are several corollaries in the gestural communication of humans and apes. First, the present data demonstrate that the mere presence of desirable but unreachable food is not sufficient to elicit gestural behavior; hence, gestures exhibited by these chimpanzees require an audience (Hostetter et al., 2001; Leavens et al., 1996). Second, there seems to be a right-hand bias for species-typical gestures in apes (Hopkins \& Cantero, 2003; Hopkins \& de Waal, 1995; Hopkins \& Leavens, 1998). Third, chimpanzees that vocalize while gesturing also gesture more with the right hand than do chimpanzees that gesture but do not vocalize (Hopkins \& Leavens, 1998). Fourth, apes in the wild point, albeit rarely (Veà \& Sabater$\mathrm{Pi}, 1998)$. A reasonable interpretation of such findings is that the behavioral capacity for intentional communication through manual gesture appeared no later than the common ancestor of humans and the great apes in the early to middle Miocene (Leavens, 2003). Because most theories of language acquisition emphasize ontogenetic continuity from nonlinguistic reference to linguistic reference in human infancy (e.g., Baldwin, 1995), these data suggest that the evolution of the human capacity for symbolic communication was perhaps predicated, in part, on the kind of coordinated manipulation of the visual attention of social agents evinced by the chimpanzees in this study (Lock \& Peters, 1996/1999).

In summary, the present study demonstrates that despite published claims to the contrary, captive chimpanzees exhibit referential communication through manual gesture with no explicit training to do so and that pointing, usually with the whole hand and less frequently with the index finger, is a frequent gestural behavior in captive representatives of this species. This finding is con- sistent with the interpretation that dependency on others to obtain otherwise unattainable objects may create a situation in which pointing emerges as an obvious solution (Leavens et al., 1996).

\section{References}

Adamson, L. R. (1996). Communication development during infancy. Boulder, CO: Westview Press.

American Psychological Association. (1992). Ethical principles of psychologists and code of conduct. American Psychologist, 47, 1597-1611.

Bakeman, R., \& Gottman, J. (1986). Observing interaction: An introduction to sequential analysis. New York: Cambridge University Press.

Baldwin, D. (1995). Understanding the link between joint attention and language. In C. Moore \& P. J. Dunham (Eds.), Joint attention: Its origins and role in development (pp. 131-158). Hillsdale, NJ: Erlbaum.

Baron-Cohen, S. (1999). The evolution of a theory of mind. In M. C. Corballis \& S. E. G. Lea (Eds.), The descent of mind: Psychological perspectives on hominid evolution (pp. 261-277). Oxford, England: Oxford University Press.

Bates, E., O'Connell, B., \& Shore, C. (1987). Language and communication in infancy. In J. Osofsky (Ed.), Handbook of infant development (pp. 149-203). New York: Wiley.

Bergmann, G. (1962). Purpose, function, scientific explanation. Acta Sociologica, 5, 225-238.

Butterworth, G. (2001). Joint visual attention in infancy. In J. G. Bremner \& A. Fogel (Eds.), Blackwell handbook of infant development (pp. 213-240). Hove, England: Blackwell.

Call, J., \& Tomasello, M. (1994). Production and comprehension of referential pointing by orangutans (Pongo pygmaeus). Journal of Comparative Psychology, 108, 307-317.

Corballis, M. C. (1991). The lopsided ape: Evolution of the generative mind. New York: Oxford University Press.

de Waal, F. B. M. (1982). Chimpanzee politics: Power and sex among apes. New York: Harper \& Row.

Gómez, J.-C. (1996). Ostensive behavior in great apes: The role of eye contact. In A. E. Russon, K. A. Bard, \& S. T. Parker (Eds.), Reaching into thought: The minds of the great apes (pp. 131-151). Cambridge, England: Cambridge University Press.

Hare, B., Call, J., Agnetta, B., \& Tomasello, M. (2000). Chimpanzees know what conspecifics do and do not see. Animal Behaviour, 59, $771-785$.

Hopkins, W. D., \& Cantero, M. (2003). From hand to mouth in the evolution of language: The influence of vocal behavior on lateralized hand use in manual gestures by chimpanzees (Pan troglodytes). Developmental Science, 6, 55-61.

Hopkins, W. D., \& de Waal, F. B. M. (1995). Behavioral laterality in captive bonobos (Pan paniscus): Replication and extension. International Journal of Primatology, 16, 261-276.

Hopkins, W. D., \& Leavens, D. A. (1998). Hand use and gestural communication in chimpanzees (Pan troglodytes). Journal of Comparative Psychology, 112, 95-99.

Hostetter, A. B., Cantero, M., \& Hopkins, W. D. (2001). Differential use of vocal and gestural communication in response to the attentional status of a human. Journal of Comparative Psychology, 115, 337-343.

Inoue-Nakamura, N., \& Matsuzawa, T. (1997). Development of stone tool use by wild chimpanzees (Pan troglodytes). Journal of Comparative Psychology, 111, 159-173.

Krause, M. A., \& Fouts, R. S. (1997). Chimpanzee (Pan troglodytes) pointing: Hand shapes, accuracy, and the role of eye gaze. Journal of Comparative Psychology, 111, 330-336.

Leavens, D. A. (2003). Integration of visual and vocal communication: Evidence for Miocene origins (Comment on Corballis). Behavioral and Brain Sciences, 26, 232-233.

Leavens, D. A., \& Hopkins, W. D. (1998). Intentional communication by 
chimpanzees: A cross-sectional study of the use of referential gestures. Developmental Psychology, 34, 813-822.

Leavens, D. A., \& Hopkins, W. D. (1999). The whole hand point: The structure and function of pointing from a comparative perspective. Journal of Comparative Psychology, 113, 417-425.

Leavens, D. A., Hopkins, W. D., \& Bard, K. A. (1996). Indexical and referential pointing in chimpanzees (Pan troglodytes). Journal of Comparative Psychology, 110, 346-353.

Leavens, D. A., Hostetter, A. B., Wesley, M. J., \& Hopkins, W. D. (2004). Tactical use of unimodal and bimodal communication by chimpanzees, Pan troglodytes. Animal Behaviour, 67, 467-476.

Lock, A., \& Peters, C. R. (1999). Editorial introduction to Part III: Ontogeny: Symbolic development and symbolic evolution. In A. Lock \& C. R. Peters (Eds.), Handbook of human symbolic evolution (pp. 371399). Oxford, England: Blackwell. (Original work published 1996)

Menzel, C. R. (1999). Unprompted recall and reporting of hidden objects by a chimpanzee (Pan troglodytes) after extended delays. Journal of Comparative Psychology, 113, 426-434.

Menzel, E. W. (1973). Leadership and communication in young chimpanzees. In E. W. Menzel (Ed.), Symposium of the IVth International Congress in Primatology. Vol. 1: Precultural primate behavior (pp. 192-225). Basel, Switzerland: Karger.

Miles, H. L. (1990). The cognitive foundations for reference in a signing orangutan. In S. T. Parker \& K. R. Gibson (Eds.), "Language" and intelligence in monkeys and apes: Comparative developmental perspectives (pp. 511-539). New York: Cambridge University Press.

Povinelli, D. J., Bering, J. M., \& Giambrone, S. (2000). Toward a science of other minds: Escaping the argument by analogy. Cognitive Science, 24, 509-541.
Povinelli, D. J., \& Davis, D. R. (1994). Differences between chimpanzees (Pan troglodytes) and humans (Homo sapiens) in the resting state of the index finger: Implications for pointing. Journal of Comparative Psychology, 108, 134-139.

Savage-Rumbaugh, E. S. (1986). Ape language: From conditioned response to symbol. New York: Columbia University Press.

Siegel, S., \& Castellan, J. (1988). Nonparametric statistics for the behavioral sciences (2nd ed.). New York: McGraw-Hill.

Thompson, N. S. (1997). Communication and natural design. In D. H. Owings, M. D. Beecher, \& N. S. Thompson (Eds.), Perspectives in ethology (Vol. 12, pp. 391-415). New York: Plenum Press.

Tomasello, M. (1995). Joint attention as social cognition. In C. Moore \& P. J. Dunham (Eds.), Joint attention: Its origins and role in development (pp. 103-130). Hillsdale, NJ: Erlbaum.

Tomasello, M., \& Call, J. (1997). Primate cognition. Oxford, England: Oxford University Press.

Veà, J. J., \& Sabater-Pi, J. (1998). Spontaneous pointing behaviour in the wild pygmy chimpanzee (Pan paniscus). Folia Primatologica, 69, 289290.

Werner, H., \& Kaplan, B. (1963). Symbol formation: An organismicdevelopmental approach to language and the expression of thought. New York: Wiley.

Woodruff, G., \& Premack, D. (1979). Intentional communication in the chimpanzee: The development of deception. Cognition, 7, 333-362.

Received August 28, 2002

Revision received June 4, 2003

Accepted June 6, 2003 